\title{
Development of a technique to evaluate the performance of cooling headers and its applications in POSCO plate mills
}

\author{
Jong-Hoon Kang ${ }^{1, a}$ and Pil-Jong Lee ${ }^{1}$ \\ ${ }^{1}$ Thermo-Fluid and Process Research Group, POSCO Technical Research Lab., Pohang 790-300, Korea
}

\begin{abstract}
Hundreds of thousands of liquid jet nozzles are widely used in controling cooling equipment to accelerate the cooling of hot plates. The holes of these nozzles become clogged or the hole size becomes smaller as foreign substances like grease, scrap, and mud containing cooling water are flushed through. In this case, cooling water sprays abnormally through the partially clogged or restricted nozzle. This causes inhomogeneous cooling of hot plates. The objective of this study is to develop an evaluation system of cooling headers including nozzles. This paper presents the method to evaluate the conditions of spraying nozzles. The sensor developed in this research measures the liquid column of spraying nozzles using differential pressure between the inside sensor block and ambient air. In addition, the results of a field test in a real, large factory are introduced in this paper.
\end{abstract}

\section{Introduction}

Slabs manufactured through a continuous casting process and a process using a reheating furnace are produced as thick plates having a predetermined thickness through roughing mill (RM) and finishing mill (FM). As shown in Figure 1, a slab is heated to a target temperature depending on the grade of steel - using a reheating furnace. The slab, having passed through the reheating furnace, is rolled to a predetermined thickness. Meanwhile, the thick plate is passed through an accelerated cooling unit so as to control grain refinement and transformation structure. It is necessary to remove factors causing the need for equipment management and non-uniformity in temperature in the rolled thick plate. The factors causing the non-uniformity in temperature are relevant to the controlling of the temperature of a material in the accelerated cooling unit. The accelerated cooling unit may be configured of several banks, and respective banks include dozens of cooling headers. A respective unit header includes thousands of nozzles forming columns of liquid and aligned in rows having

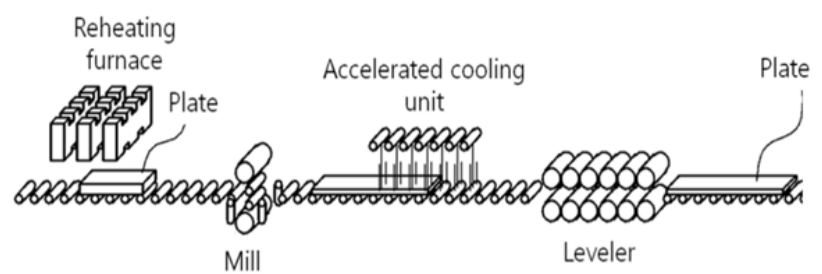

uniform intervals.

Figure 1. Schematic diagram of the thick plate manufacturing process.

${ }^{\mathrm{a}}$ Corresponding author: engdr@posco.com
An impinging jet is a flow state that is widely prevalent in nature, such as the flow escaping from a water tap system, the falls of a waterfall from a high mountain, and so on. Because of high-efficiency heat transfer, impinging jets have been widely used to cool high temperature products in modem industrial production. For example, air impinging jets can be used to cool high temperature electronic elements in the electronics industry; water impinging jets can be used to accelerate the cooling of steel plates in the steel rolling process to achieve better mechanical properties and to improve productivity; likewise, impinging jet cooling has been widely used in the nuclear industry and the glass industry for a long time. However, just like the jet breakup of a tap water stream, jet breakup phenomenon is possible after the liquid impinging jet exited from the Accelerated Control Cooling (ACC) equipment had passed a certain.

The stability parameter is used to characterize jet breakup. Depending upon the point of view that is taken, can be characterized as the reciprocal of the amplification rate for disturbances or as the logarithm of the initial disturbance level. The stability of liquid jets has been studied for more than a century as a consequence of important practical applications such as cooling processes as well as fuel injection systems(citation needed). A number of experimental studies have been run to understand the mechanisms of the disintegration of liquid jets into droplets (citation needed). The measurement methods employed to study the stability of liquid jets allow us to classify them into two types that we will refer 
to as the local type and global type. Local type measurements are concerned with the temporal evolution of the interfacial displacement of the jet at a particular downstream position. These techniques commonly use a laser beam interacting with a small portion of the liquid jet.

Diffracted or transmitted light is analyzed to determine the diameter of the jet. For the global type measurement, the entire jet, or a significant part of it, is spatially analyzed. Global type techniques use imaging systems which record a shadow of the jet. Manual or automatic image analysis is used to determine the downstream variation of the jet diameter. Most experiments dealing with the measurement of the rate of growth of the disturbance use controlled jet destabilization.

The nozzles provided in the headers of the accelerated cooling unit are easily blocked in the inner portions of nozzles and outer portions thereof (jet holes) due to a difference in the ionization of constituent components thereof, heating and cooling. The blocking phenomenon in the nozzles of headers mostly occurs as holes are plugged with scales generated due to degradation. Therefore, in a case in which only portions of a considerable amount of nozzles in one unit header are blocked or nozzles are narrowed due to other foreign matter, corrosion, or the like, the cooling of the thick plate to its finish cooling temperature (FCT) may be difficult to attain or a cooling defects may be caused. Furthermore, when the nozzles are blocked or narrowed, internal pressure of the accelerated cooling unit increases and relevant components connected thereto, such as pipes, measuring instruments, pumps, and the like, are more apt to break down.

In this study, we developed the POCHEF (POSCO Cooling Header Evaluation Facility) for determining the condition of the nozzle using differential pressure between the inside sensor block and ambient air. In addition, the results of a field test in a real, large factory in POSCO are introduced in this paper.

\section{Experimental methods}

The spraying pattern varied with the condition of nozzles inside the cooling header. As shown in Figure 2, the spraying patterns can be classified into three types. If strange substances are adhered to the nozzle hole or the machining condition of nozzles are wrong, the liquid column of the impinging jet tilts forward-backward/ leftright. In addition, the cooling water does not spray if the nozzle is clogged. Figure 3 shows the condition of liquid column spraying at the cooling header in large factory.

It was observed prior to this study that the impinging pressure of the abnormal jet is lower than that of the normal jet. The collision force of the column of liquid indicates whether the column of liquid is normally dispensed through the nozzles, or whether the nozzles are blocked partially or in the entirety thereof so as not to allow the column of liquid to be dispensed in a normal amount. Alternatively, measurement values recorded by the pressure sensor may differ from one another depending on an extent of collision force which the column of liquid itself collides with the membrane member. In this study, the cooling header evaluation system was designed and made the pilot equipment using the principles of this phenomenon. Pilot equipment was installed in the cooling header used in the field.

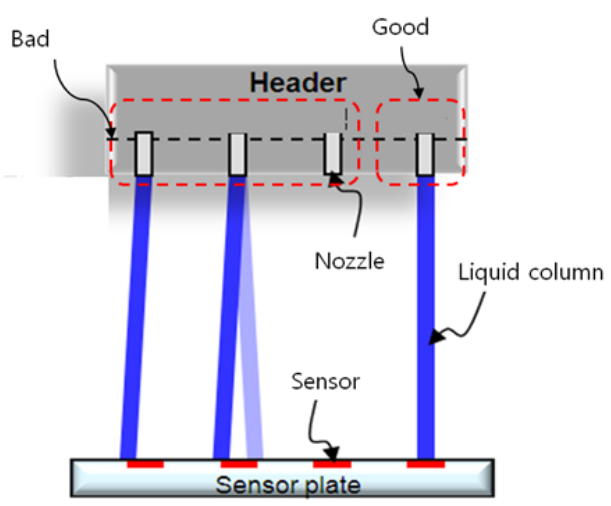

Figure 2. Schematic diagram of liquid column according to nozzle condition of multi-jet cooling header.

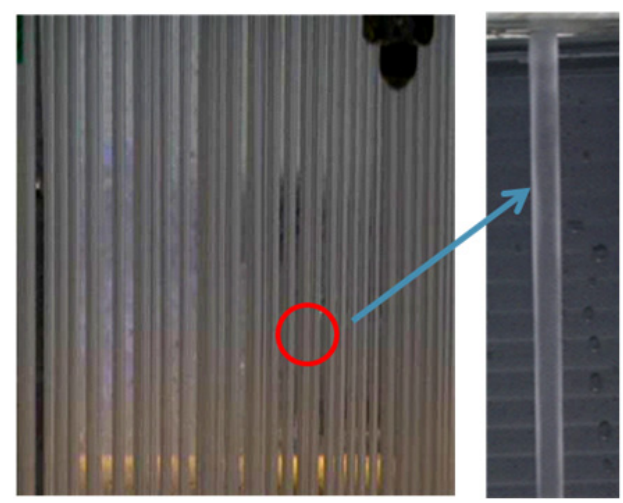

(a) Good

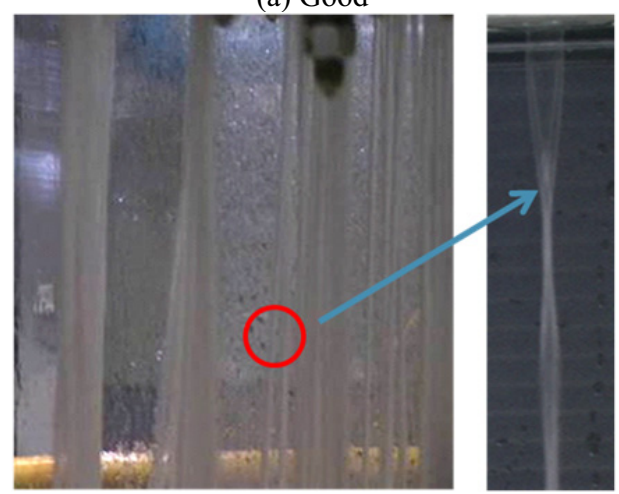

(b) $\mathrm{Bad}$

Figure 3. Comparative photographs of the liquid column condition of the impinging jet.

A sensor device may include a housing including an air space; a membrane member provided with the housing and inducing a change in a volume of the air space at the time of a collision or contact of a pressure-applying material with the membrane member; and a sensor unit provided in the housing to communicate with the air space. The pressure sensor may measure a pressure value and then generate a voltage signal. The diameter of sensor membrane is $18 \mathrm{~mm}$. The sensor block has 12 sensors and 2 array. Figure 4 shows the schematic diagram of the sensor block. 
An apparatus for evaluating performance of a cooling system, including a sensor device, may include: a sensor mounting unit provided on a movement path on which the cooling target material is moved and provided with a cooling header including nozzles, and the sensor device described above, provided in the sensor mounting unit to be aligned to correspond to the nozzles. The sensor structure allows for the generation of a quantitative signal as well as a qualitative signal corresponding to pressure applied at the time of contact of the liquid column, injection water dispensed or injected through nozzles, or the like, and allow for the establishment of data.

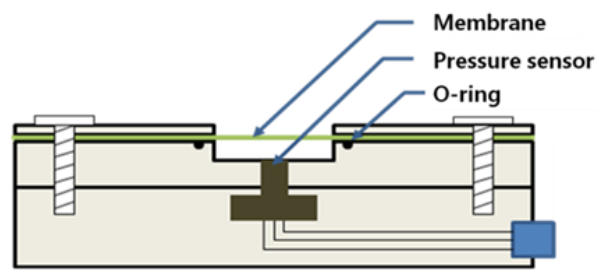

Figure 4. Schematic diagram of the sensor.

Figure 5 shows the schematic diagram of the measurement method using the cooling header evaluation system. The cooling jet system has 1,000 nozzles with nozzle hole $5 \mathrm{~mm}$ in diameter. Total injection flow rate is $6 \mathrm{~m}^{3} / \mathrm{min}$ per one cooling header; each nozzle $61 / \mathrm{min}$. The moving speed of the sensor plate is $1 \sim 3 \mathrm{~m} / \mathrm{min}$. When the column of liquid dispensed from the upper header falls on and collides with the sensor, the water flow increases, and the pressure sensor may measure a pressure value (generate a voltage signal). This system measured automatically all of the nozzles in the width direction of the cooling header. The wireless transmission and reception system was used for transmitting measured data.

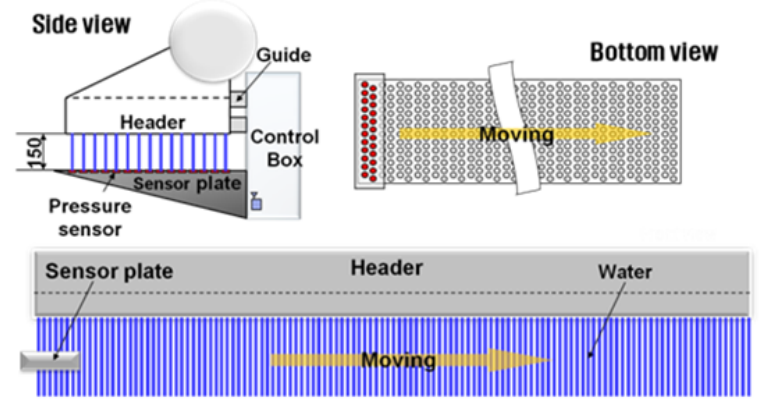

Figure 5. Schematic diagram of of the measurement method using the cooling header evaluation system.

\section{Results and discussion}

The membrane of the sensor device is formed using a material appropriate such as urethane, silicon, stainless steel, or the like, and may have a thickness appropriate in a range of from 0.5 to $1.5 \mathrm{~mm}$. When the membrane has a reduced thickness of less than $0.5 \mathrm{~mm}$, although reaction sensitivity of a sensor may be excellent, durability thereof may be decreased, and when the membrane has a thickness greater than $1.5 \mathrm{~mm}$, although the durability thereof may be secured, a deflection amount of the membrane at the time of the generation of collision by the pressure-applying material may be relatively small to reduce a reaction speed or the sensitivity thereof. In addition, when the thickness of the membrane is less than $0.5 \mathrm{~mm}$, the lifespan of the membrane or level of elasticity to return to its original position after the collision of the column of liquid may be decreased, such that the overall lifespan may be reduced. Meanwhile, in consideration for securing durability in terms of repetitive collisions of the column of liquid and the return of the membrane to the initial position after the collision of the column of liquid, the membrane may be formed using a stainless material.

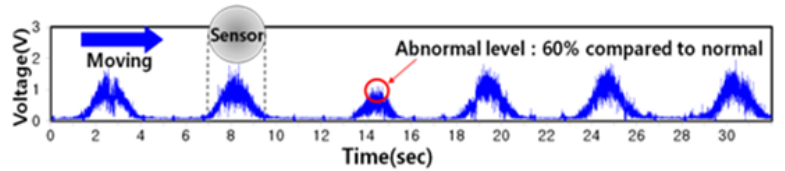

Figure 6. Analyzed data of the nozzle condition gathered by the POCHEF sensor.

Numerous data received through the POCHEF was processed in a short period of time. In addition, the optimal algorithm was necessary for removing noise caused by auxiliary actuating devices and water cooling installations in the factory building. In this case, when a reference pressure value indicating that pressure is not being applied to sensors is preset and a controlling unit may compare differential pressure with the measured pressure value, quantitatively measured data may also be established. Figure 6 shows the measured voltage signal. When the column of liquid (Figure 2 "Good") is normally dispensed from the upper header, the column of liquid continuously collides with the membrane of the sensor device. This may indicate that an electrical output signal generated by the pressure sensor may be represented the normal level of nozzles. However, when portions of the nozzles of the header are blocked, so as not to allow for the normal dispensing of liquid and columns of discontinuous liquid (Figure 2 "Bad") are dispensed, a voltage signal transferred by the pressure sensor may be short-circuited(ex. abnormal level in Figure 6).

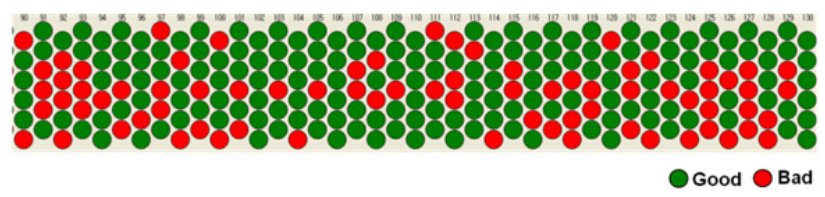

Figure 7. The measurement result using POCHEF in field.

The POCHEF may evaluate the column of normal liquid and the column of abnormal liquid dispensed through the nozzles of the cooling header. The extracted data of the field test result is illustrated in Figure 7. The green circle of the legend means the normal nozzle and the red one means the abnormal nozzle. From this results, the condition of cooling header can be evaluated. In addition, the cooling header may be frequently monitored using the POCHEF.

\section{Conclusions}

To improve maintainability and operability of cooling systems, we investigated a sensor device and an 
apparatus for evaluating the performance of a cooling system, and more particularly, a sensor device allowing for the generation of a quantitative signal as well as a qualitative signal corresponding to the pressure of liquid impacting a membrane member. The pressure of a column of water injected from a nozzle on a membrane member was measured allowing for the establishment of data and preventing the occurrence of errors in measurement due to changes in temperature, to precisely evaluate cooling system performance (in a pouring or injection state) at least quantitatively.

It is necessary to provide a sensor device allowing for the establishment of a sensor to generate a quantitative signal as well as a qualitative signal corresponding to pressure applied to a membrane component of the sensor at the time of collision (contact) of pressure-applying matter such as liquid, a column of liquid, or injection water dispensed or injected through a nozzle. A sensor device capable of preventing the occurrence of measurement errors by controlling a temperature of air transferring pressure applied to a sensor, to allow gas density to constantly change depending on coolant or ambient atmospheric temperature, and secure precision in measurement is required.

It is also necessary to provide a sensor device allowing for stabilized and quantitative evaluation by filling an operation space with incompressible fluid in which no change or a relatively small change in density according to temperature occurs, so as not to be affected by temperature.

Therefore, the POCHEF provides an apparatus for evaluating cooling system performance, capable of preventing the occurrence of cooling errors through quantitative, rapid and precise evaluation of cooling system performance, based on the sensor device according to the present disclosure, and allowing for easy maintenance and repair of equipment by determining the state of nozzles of a cooling system, based on automation in a poor environment confined to a relatively small workplace.

\section{References}

1. Lord Rayleigh, On the instability of jets, [in] Proc. 10th Math. Soc., London, (1878)

2. C. Weber, Zum zerfall eines flüssigkeitsstrahles, Z. Angew. Math. Mech., 11(1931)

3. D.B. Bogy, Drop formation in a circular liquid jet, Annu. Rev. Fluid Mech., 11(1979)

4. L. Crane, S. Birch, and P.D. McCormack, The effect of mechanical vibration on the breakup of a cylindrical water jet in air, Br. J. Appl. Phys., 15(1964)

5. R.J. Donnelly and W. Glaberson, Experiment on capillary instability of a liquid jet, [in] Proc. R. SOC., A290(1966)

6. E.F. Goedde and M.C. Yuen, Experiments on liquid jet instability, J. Fluid Mech, 40(1970)

7. A.L. Yarin, Free Liquid Jets and Films: Hydrodynamics and Rheology, Longman Scientific and Technical, Harlow, Essex, (1993)
8. P.-K. Wu, R.F. Miranda, and G.M. Faeth, Effects of initial flow conditions on primary breakup of nonturbulent and turbulent round liquid jet, At. Sprays, 5(1995)

9. Z. Dai, W.-H. Chou, and G.M. Faeth, Drop formation due to turbulent primary breakup at the free surface of plane liquid wall jets, Phys. Fluids, 10(1998)

10. T.A. Kowalewski, On the separation of droplets from a liquid jet, Fluid Dyn. Res., 17(1996)

11. A.W. Kerst, B. Judat, and E.-U. Schlunder, Flow regimes of free jets and falling films at high ambient pressure, Chem. Eng. Sci., 55(2000)

12. K.A. Sallam, Z. Dai, and G.M. Faeth, Liquid breakup at the surface of turbulent round liquid jets in still gases, Int. J. Multiphase Flow, 28(2002) 